\title{
Tuberculose em indígenas da Amazônia brasileira: estudo epidemiológico na região do Alto Rio Negro
}

\author{
Diana Patrícia Giraldo Rios, ${ }^{1}$ Jocieli Malacarne, ${ }^{1}$ \\ Luiz Carlos Corrêa Alves, ${ }^{1}$ Clemax Couto Sant'Anna, ${ }^{2}$ \\ Luiz Antonio Bastos Camacho e Paulo Cesar Basta ${ }^{1}$
}

Como citar

Rios DPG, Malacarne J, Alves LCC, Sant'Anna CC, Camacho LAB, Basta PC. Tuberculose em indígenas da Amazônia brasileira: estudo epidemiológico na região do Alto Rio Negro. Rev Panam Salud Publica. 2013:33(1):22-29.

RESUMO Objetivo. Descrever as características sociodemográficas e clínico-epidemiológicas dos casos de tuberculose notificados na população indígena do Município de São Gabriel de Cachoeira, Estado do Amazonas, Brasil, e identificar fatores associados ao óbito durante o tratamento; e estimar a prevalência de infecção latente por tuberculose (ILTB) e fatores associados e obter informações sobre o itinerário terapêutico e a percepção acerca do adoecimento dos indígenas do Distrito de Iauaretê.

Métodos. Primeiramente, realizou-se um estudo epidemiológico retrospectivo (1997 a 2007) com base nos dados do Sistema de Informação de Agravos de Notificação. Posteriormente, realizou-se um estudo transversal (2010) em sintomáticos respiratórios e contatos no Distrito Indígena de Iauaretê.

Resultados. Registraram-se 723 casos novos, com incidência de 273,4/100 000 e mortalidade de 13,2/100 000. Houve predominio em homens (57\%), idade $>45$ anos (37,6\%), pessoas sem escolaridade $(42,7 \%)$ e na área rural (76,9\%). Doentes de 0 a 20 anos apresentaram menor chance de óbito quando comparados à idade $\geq 45$ anos $(O R=0,3 ;$ IC95\%:0,1 a 0,9). Em Iauaretê, que concentrou 15,3\% das notificações, foram entrevistados 184 indígenas. Revelou-se prevalência de ILTB de 76,1\%. Prova tuberculinica $\geq 5 \mathrm{~mm}$ associou-se com idade $\geq 15$ anos, história de tuberculose ativa e alterações radiológicas. Tuberculose anterior foi citada por 54 indígenas (29,3\%). A principal explicação para o adoecimento foi sopro/envenenamento $(24,1 \%)$. O itinerário terapêutico incluiu medicamentos industrializados (42,6\%), plantas medicinais/ raízes, pajelança/xamanismo e reza $(42,7 \%)$.

Conclusões. A prevalência de infecção tuberculosa e doença ativa nessa população foi elevada. As estratégias de controle tiveram resultados positivos, com redução na incidência em tempos recentes. Entretanto, para controlar a tuberculose é necessário intensificar a vigilância dos contatos e aperfeiçoar as estratégias de comunicação das equipes com a população indígena.

Palavras-chave Tuberculose; índios sul-americanos; saúde de populações indígenas; epidemiologia; sistemas de saúde; Brasil.

1 Escola Nacional de Saúde Pública (ENSP), Fundação Oswaldo Cruz (FIOCRUZ), Rio de Janeiro (RJ), Brasil. Correspondência: Paulo Cesar Basta, paulobasta@gmail.com

2 Universidade Federal do Rio de Janeiro (UFRJ), Rio de Janeiro (RJ), Brasil.
A cada ano no Brasil são notificados aproximadamente 80000 casos novos de tuberculose $(1,2)$. Assim como em outras partes do mundo, a doença está associada às precárias condições de vida da população. Sua distribuição não é homogênea, havendo concentração de casos em grupos vulneráveis, tais como moradores de rua, presidiários e minorias étnicas (2-4). Entre esses 
grupos vulneráveis destacam-se os indígenas da região amazônica, com incidências de tuberculose que ultrapassam $1000 / 100000$ habitantes (5-9), enquanto as médias nacionais situam-se na faixa de 40/100 000 (10).

O Estado do Amazonas concentra o maior número de indígenas no país, 168680 indivíduos, segundo o último censo nacional (11). São Gabriel da Cachoeira, situado na fronteira com a Colômbia e a Venezuela, é o município com a maior concentração de indígenas no Brasil - dos 37896 habitantes recenseados em 2010, $29017(76,6 \%)$ se autodeclararam indígenas, pertencentes a 23 etnias $(11,12)$. Face à importante densidade populacional de indígenas e à alta mobilidade desses grupos nas áreas de fronteira, vários pesquisadores têm trabalhado para ampliar a compreensão sobre aspectos epidemiológicos da tuberculose na região.

Analisando os livros de registro das unidades hospitalares entre 1977 e 1996, Buchillet e Gazin (13) descreveram incidências de tuberculose acima de 200/100000 na população indígena no noroeste amazônico. Em períodos mais recentes, outros autores $(14,15)$ revelaram, do mesmo modo, incidências superiores a 280/100000, ratificando a gravidade da tuberculose nessa região. Entretanto, permanecem lacunas no conhecimento quanto aos resultados dos tratamentos instituídos, às características operacionais de controle da enfermidade, aos fatores associados à infecção latente por tuberculose (ILTB) e aos aspectos culturais relativos à doença.

Nosso objetivo foi descrever as características sociodemográficas e clínicoepidemiológicas dos casos de tuberculose notificados na população indígena do Município de São Gabriel de Cachoeira e identificar fatores associados ao óbito durante o tratamento no período de 1997 a 2007. Buscamos também examinar uma amostra de sintomáticos respiratórios (SR) e contatos, visando a estimar a prevalência de ILTB e fatores associados e a obter informações sobre o itinerário terapêutico e a percepção acerca do adoecimento dos indígenas do Distrito de Iauaretê.

\section{MATERIAIS E MÉTODOS}

Esta pesquisa contemplou dois componentes: um estudo retrospectivo (des- crição das características sociodemográficas e clínico-epidemiológicas dos casos de tuberculose) e um estudo transversal (estimativa da prevalência de ILTB, associações com as respostas à prova tuberculínica (PT), itinerário terapêutico e percepção acerca do adoecimento nos indígenas de Iauaretê).

São Gabriel da Cachoeira situa-se no extremo noroeste do Estado do Amazonas ( $\left.0^{\circ} 7^{\prime} 48^{\prime \prime} \mathrm{S}, 67^{\circ} 5^{\prime} 20^{\prime \prime} \mathrm{W}\right)$, na margem esquerda do Rio Negro. Faz fronteira ao norte com a Colômbia e a Venezuela e ao sul e leste com Santa Isabel do Rio Negro (16). O município encontra-se a $852 \mathrm{~km}$ de Manaus, capital do Estado do Amazonas, com acesso apenas por via fluvial (51 horas de barco) e aérea (4 horas de voo).

O segundo maior polo de concentração humana em São Gabriel da Cachoeira é o Distrito Indígena de Iauaretê, situado no médio Rio Uaupés, na fronteira com a Colômbia. Em 2009, a região somava 2903 habitantes, com discreto predomínio de homens $(51 \%)$ e fração expressiva $(36 \%)$ de crianças e adolescentes com menos de 15 anos (17).

Segundo Andrello (18), Iauaretê, conhecido como "Cidade do Índio", constitui caso único em concentração populacional, pois agrega 12 comunidades indígenas, com 15 etnias. Atualmente, a organização social em Iauaretê se caracteriza pela presença de residências de famílias nucleares, intercaladas por centros comunitários e pequenos comércios, igrejas, um pelotão especial de fronteira do exército, uma unidade geradora de energia termoelétrica, uma unidade hospitalar, uma agência dos correios e um banco postal. Essa estrutura faz do local uma referência social, econômica e cultural na região.

\section{Estudo de coorte retrospectivo}

O objetivo deste componente foi descrever as características sociodemográficas e clínico-epidemiológicas dos casos de tuberculose notificados e identificar fatores associados ao óbito durante o tratamento no período de 1997 a 2007. Os casos notificados foram obtidos do Sistema de Informação de Agravos de Notificação (SINAN), do Sistema de Informação da Atenção à Saúde Indígena (SIASI) e do livro de registro de casos do Distrito Sanitário Especial Indígena do Alto Rio Negro (DSEI-ARN). Foram incluídos todos os casos novos disponíveis nas bases consultadas e excluídos registros duplicados, aqueles com situação de encerramento (mudança de diagnóstico) e os ocorridos em não indígenas.

Para identificar os casos em indígenas, foi realizada consulta à variável raça/cor constante na ficha de notificação. Essa variável é definida por autodeterminação, de acordo com os atributos adotados pelo IBGE (19), ou seja: o próprio doente se classifica como branco, preto, amarelo, pardo ou indígena.

Os casos foram analisados segundo as variáveis sexo, faixa etária, local de residência (urbano ou rural), escolaridade (anos de estudo), forma clínica (pulmonar, extrapulmonar e mista), exames empregados para o diagnóstico (baciloscopia e cultura de escarro, radiografia de tórax e PT, baciloscopias de controle do $2^{\circ}, 4^{\circ}$ e $6^{\circ}$ mês, tratamento supervisionado, exames de contato e situação de encerramento.

Para o cálculo dos coeficientes de incidência, utilizaram-se no numerador os casos novos e, para os coeficientes de mortalidade, os casos que tiveram como desfecho o óbito, independentemente da causa. No denominador, foram utilizados dados populacionais sobre o contingente populacional indígena provenientes dos censos demográficos nacionais (2000 e 2010) (http://www.sidra.ibge. gov.br). Para as estimativas populacionais intercensitárias, foi utilizado o método da progressão geométrica, com taxa média de crescimento de $2,4 \%$ ao ano.

\section{Estudo transversal para investigar sintomáticos respiratórios e contatos}

No segundo componente, foi analisada amostra não probabilística selecionada pela equipe multidisciplinar de saúde. No período estudado foram selecionados 130 adultos e crianças com sintomas respiratórios (tosse seca ou produtiva, dispneia e febre) e outros 54 com história de tratamento anterior para tuberculose. Por solicitação da equipe do DSEI-ARN, nosso grupo de pesquisa realizou avaliação clínica daqueles indivíduos. Os indígenas selecionados foram entrevistados por meio de questionário semiestruturado que contemplou as variáveis sexo, faixa etária, renda, escolaridade, número de pessoas/domicílio, história anterior de tratamento para tuberculose, contato com doente de tuberculose e cicatriz vacinal de BCG. 
Aqueles que referiram história anterior de tuberculose foram interrogados sobre o número de episódios da doença, resultados do tratamento e itinerário terapêutico adotado. Por meio de uma pergunta aberta, buscou-se conhecer a percepção dos indivíduos sobre o adoecimento por tuberculose.

Para esta investigação, realizaramse procedimentos normatizados pelo Programa Nacional de Controle da Tuberculose (PNCT). Independentemente dos antecedentes vacinais e da idade, todos os sujeitos foram submetidos a avaliações clínicas e PT com aplicação intradérmica de $0,1 \mathrm{~mL}$ (2 UT) da tuberculina PPD RT 23 (Staten Serum Institute, Copenhague, Dinamarca) no terço médio da face anterior do antebraço esquerdo (20).

Foram oferecidos aos SR coletores universais para obtenção de duas amostras de escarro (uma no momento da entrevista e outra na manhã seguinte, em jejum). As amostras foram fixadas e coradas pelo método de Ziehl Neelsen para realização da baciloscopia e semeaduras em meio de Ogawa-Kudoh na própria localidade. Os meios semeados foram encaminhados ao laboratório do Núcleo de Doenças Infecciosas da Universidade Federal do Espírito Santo (unidade de referência nacional), onde foram incubados em estufa a $37^{\circ} \mathrm{C}$ até obtenção dos resultados.

Adicionalmente, foram realizadas radiografias de tórax com equipamento portátil, adaptado às condições adversas das aldeias, em incidência póstero-anterior (PA) nos indígenas selecionados. As anormalidade nas imagens em PA foram investigadas também em perfil. Um dos autores emitiu laudos padronizados segundo Den Boon et al. (21).

Os dados foram armazenados em planilhas eletrônicas e analisados no programa Statistical Package for the Social Sciences (SPSS), versão 9.0. O teste do qui-quadrado $\left(\chi^{2}\right)$ foi utilizado para a comparação de proporções. Considerouse um nível de significância estatística de 5\%. A análise de regressão logística múltipla foi precedida de testagem e seleção por meio de análise univariada entre a variável dependente óbito e as variáveis que se mostraram associadas em nível de $20 \%$.

O modelo de regressão logística múltipla foi ajustado, seguindo o método stepwise forward, sendo as variáveis de interesse introduzidas em ordem decres- cente de significância estatística. Permaneceram no modelo final as variáveis que se mostraram significativas em nível de $5 \%$ e que foram utilizadas como variáveis de controle. A razão de chances (odds ratio, OR) foi utilizada como medida de associação.

A presente pesquisa foi aprovada pelo Comitê de Ética em Pesquisa da Escola Nacional de Saúde Pública (parecer 146/10); pelo Departamento de Saúde Indígena da Fundação Nacional de Saúde (FUNASA); pela Chefia do DSEI-ARN; pela Secretaria Municipal de São Gabriel da Cachoeira; e pelo Conselho Distrital de Saúde Indígena do Alto Rio Negro. Os procedimentos relativos ao estudo transversal foram realizados somente após esclarecimento e assinatura, pelos participantes, do termo de consentimento livre e esclarecido.

\section{RESULTADOS}

Originalmente, o conjunto disponível para análise, considerando todas as bases de dados consultadas, continha 854 notificações para a população residente em São Gabriel da Cachoeira no período de 1997 a 2007. Desse total, foram excluídas 16 (1,9\%) duplicidades, 52 (6,1\%) casos que não eram novos, 36 (4,2\%) casos com mudança de diagnóstico no encerramento e $27(3,2 \%)$ casos em não indígenas, restando, portanto, 723 casos para análise.

Foi mais frequente a forma clínica pulmonar $(85,8 \%)$. Houve maior concentração de casos em homens (57\%), em maiores de 45 anos $(37,6 \%)$, em pessoas sem escolaridade $(42,7 \%)$ e na área rural (76,9\%). Em relação aos casos oriundos da área rural $(\mathrm{n}=556)$, Iauaretê concentrou 15,3\% (85/556) das notificações, seguido das aldeias Maturacá e Maiá, onde residem, na fronteira com a Venezuela, os Ianomâmi, com 10,3\% (57/556) e 5,6\% (31/556) dos casos, respectivamente.

Com exceção da radiografia de tórax, que foi registrada em 78,0\% (564/723) dos casos, a maioria dos exames complementares de diagnóstico foi subutilizada. $\mathrm{O}$ percentual de positividade das baciloscopias de escarro foi $49,5 \%$ (209/422) e 33,3\% (12/36) nos maiores e menores de 15 anos, respectivamente. A PT foi empregada em 35,1\% (254/723) e a cultura de escarro, em 2,5\% (18/723) das notificações.

$\mathrm{O}$ regime de tratamento supervisionado foi informado em 15,5\% dos casos.
As baciloscopias de escarro do $2^{\circ}, 4^{\circ}$ e $6^{\circ}$ mês de tratamento não foram realizadas em mais de $85 \%$ dos tratamentos. O exame de contatos não foi realizado em $53 \%$ das notificações (383/723).

A análise global da situação de encerramento revelou que em $86,2 \%$ das notificações o desfecho foi "cura", em 9,0\% foi "abandono" e em 4,1\% foi "óbito". Entretanto, a proporção de óbitos (letalidade) foi expressivamente maior entre os casos de recidiva (3/18) e reingresso após abandono (1/6), totalizando $16,7 \%$. Não foram reportados casos multirresistentes no período.

O coeficiente médio de mortalidade estimado para a população indígena de São Gabriel da Cachoeira foi de 13,24/100 000, variando de 0,0 em 2007 a 38,45 por 100000 em 2001 (tabela 1). Segundo o modelo logístico, doentes com idade de 0 a 20 anos apresentaram menor chance de óbito quando comparados aos com $\geq 45$ anos (OR =0,3; IC95\%: $0,1$ a 0,9$)$ (tabela 2).

Após atingirem níveis máximos em 2001, os coeficientes de incidência em São Gabriel da Cachoeira diminuíram substantivamente ao longo do período. Apesar da flutuação, o mesmo foi observado em Iauaretê (tabela 1).

\section{Estudo transversal}

No inquérito em Iauaretê foram avaliados 184 indígenas. A média de idade foi de 41,2 anos (desvio padrão: 21,2) e 31 indivíduos $(16,8 \%)$ tinham menos de 15 anos. Houve predomínio de mulheres $(52,2 \%)$, de sujeitos que informaram como grau de instrução pelo menos o ensino fundamental incompleto $(86,8 \%)$, renda mensal maior ou igual a um salário mínimo $(60,4 \%)$ e residência em domicílio com seis ou mais indivíduos $(57,2 \%)$.

Quarenta e nove indígenas referiram tosse no momento da entrevista, tendo sido coletadas amostras de escarro em 39. Realizaram-se 61 baciloscopias e semeaduras em meio de Ogawa-Kudoh, sendo todas as culturas negativas para $\mathrm{Myco-}$ bacterium tuberculosis. Quatro culturas (do mesmo indivíduo) foram positivas para Klebsiella pneumoniae. Não houve crescimento de outras micobácterias.

Foram realizadas 164 radiografias de tórax, das quais 36 (22\%) apresentaram alterações, com predomínio de nódulos calcificados e traves fibróticas em 30 imagens, consideradas sequelares. Em seis radiografias foram identificados in- 
TABELA 1. Distribuição anual dos casos novos notificados, incidência e mortalidade da tuberculose em indígenas de São Gabriel da Cahoeira e incidência no Distrito Indígena de lauaretê (AM), Brasil, 1997 a 2007

\begin{tabular}{|c|c|c|c|c|c|c|c|c|}
\hline \multirow[b]{3}{*}{ Ano } & \multicolumn{5}{|c|}{ São Gabriel da Cachoeira } & \multicolumn{3}{|c|}{ Distrito de lauaretê } \\
\hline & \multirow[b]{2}{*}{ População } & \multicolumn{2}{|c|}{ Incidência } & \multicolumn{2}{|c|}{ Mortalidade } & \multirow[b]{2}{*}{ População } & \multicolumn{2}{|c|}{ Incidência } \\
\hline & & Casos & Taxa & Óbitos & $\begin{array}{c}\text { Taxa } \\
(\text { por } 100000)\end{array}$ & & Casos & Taxa \\
\hline 1997 & 21273 & 81 & 380,76 & 4 & 18,80 & 2184 & 6 & 274,73 \\
\hline 1998 & 21787 & 64 & 293,75 & 2 & 9,18 & 2236 & 18 & 804,87 \\
\hline 1999 & 22314 & 57 & 255,45 & 1 & 4,48 & 2290 & 14 & 611,34 \\
\hline 2000 & 22853 & 71 & 310,68 & 3 & 13,13 & 2345 & 6 & 255,86 \\
\hline 2001 & 23405 & 105 & 448,62 & 9 & 38,45 & 2401 & 17 & 708,04 \\
\hline 2002 & 23971 & 100 & 417,17 & 2 & 8,34 & 2459 & 9 & 366,00 \\
\hline 2003 & 24550 & 82 & 334,01 & 9 & 36,66 & 2518 & 6 & 238,28 \\
\hline 2004 & 25144 & 60 & 238,63 & 2 & 7,95 & 2578 & 2 & 77,58 \\
\hline 2005 & 25751 & 49 & 190,28 & 2 & 7,77 & 2640 & 4 & 151,52 \\
\hline 2006 & 26374 & 27 & 102,37 & 1 & 3,79 & 2704 & 3 & 110,95 \\
\hline 2007 & 27011 & 27 & 99,96 & 0 & 0,00 & 2769 & 0 & 0,00 \\
\hline Total & 264433 & 723 & 273,42 & 35 & 13,24 & 27124 & 85 & 313,38 \\
\hline
\end{tabular}

Fonte: Sistema de Informação de Agravos de Notificação (SINAN), Secretaria Municipal de Saúde (SEMSA), São Gabriel da Cachoeira (AM), Brasil (1997 a 2007); Instituto Brasileiro de Geografia e Estatística (IBGE) (1991, 2000, 2010).

TABELA 2. Fatores associados ao óbito na vigência do tratamento (independentemente da causa) entre os casos novos de tuberculose, São Gabriel da Cachoeira (AM), Brasil, 1997 a 2007

\begin{tabular}{|c|c|c|c|}
\hline Variáveis explicativas & No. & OR bruta (IC80\%) & OR ajustada (IC95\%) \\
\hline \multicolumn{4}{|l|}{ Faixa etária (anos) } \\
\hline$\geq 45$ & 272 & 1,0 & 1,0 \\
\hline $0-20$ & 217 & $0,4(0,2$ a 0,7$)$ & $0,3(0,1$ a 0,9$)$ \\
\hline 20-45 & 234 & $0,5(0,3$ a 0,9$)$ & $0,6(0,2$ a 1,3$)$ \\
\hline \multicolumn{4}{|l|}{ Forma clínica } \\
\hline Pulmonar & 620 & 1,0 & 1,0 \\
\hline Extra-pulmonar & 103 & $0,2(0,1$ a 0,7$)$ & $0,2(0,2$ a 1,4$)$ \\
\hline \multicolumn{4}{|l|}{ Zona de residência } \\
\hline Rural & 554 & 1,0 & 1,0 \\
\hline Urbana & 166 & $1,5(0,9$ a 2,5$)$ & $1,2(0,5$ a 2,8$)$ \\
\hline \multicolumn{4}{|l|}{ Sexo } \\
\hline Masculino & 412 & 1,0 & 1,0 \\
\hline Feminino & 311 & $1,0(0,7$ a 1,6$)$ & $1,0(0,5$ a 2,1$)$ \\
\hline \multicolumn{4}{|l|}{ Baciloscopia } \\
\hline Positiva & 221 & 1,0 & 1,0 \\
\hline Negativa & 237 & $0,8(0,5$ a 1,5$)$ & $0,9(0,4$ a 2,3$)$ \\
\hline Não realizada & 265 & $0,9(0,5$ a 1,6$)$ & $1,7(0,7$ a 4,4$)$ \\
\hline
\end{tabular}

Fonte: Sistema de Informação de Agravos de Notificação (SINAN), Secretaria Municipal de Saúde (SEMSA), São Gabriel da Cachoeira (AM).

filtrados e/ou condensações e/ou derrame pleural sugestivos de tuberculose ativa e/ou outra doença pulmonar.

As reações à $\mathrm{PT} \geq 5 \mathrm{~mm}$ estiveram associadas à idade $\geq 15$ anos, antecedentes de tuberculose e alterações radiográficas sugestivas de sequela ou tuberculose ativa (tabela 3 ). A prevalência de ILTB foi estimada em $76,1 \%$. A mediana das reações foi $14 \mathrm{~mm}$ e o desvio padrão, 7,4 $\mathrm{mm}$ (figura 1). Dos 31 indivíduos que não apresentaram reações à PT (zero $\mathrm{mm}), 18(58 \%)$ tinham $<15$ anos.

Tratamento anterior foi referido por 54 entrevistados $(29,8 \%)$, sendo que sete adoeceram duas vezes. A principal ex- plicação para o adoecimento foi "sopro e/ou envenenamento" (24,1\%; 13/54). Em $42,6 \%$ dos relatos o itinerário terapêutico inicial incluiu medicamentos da farmácia/posto de saúde. Entretanto, em $42,7 \%$ dos tratamentos, foi relatado o uso de plantas medicinais/raízes, pajelança/ xamanismo e reza. Quanto ao resultado do tratamento, 46 sujeitos $(85,2 \%)$ referiram cura e seis abandono, sendo a melhora dos sintomas a principal causa da interrupção (tabela 4).

Não foram identificados casos de tuberculose ativa na amostra investigada. Entretanto, 11 casos de ILTB e um caso de pneumonia bacteriana foram encami- nhados para tratamento no serviço local de saúde.

\section{DISCUSSÃO}

A análise dos casos de tuberculose notificados em indígenas em São Gabriel da Cachoeira confirmou coeficientes de incidência e de mortalidade elevados, porém decrescentes ao longo do período. $\mathrm{O}$ estudo transversal no Distrito de Iauaretê revelou ainda que, independentemente da idade e da situação vacinal, o risco de infecção tuberculosa e doença ativa nessa população foi elevado. Apesar de as imagens radiológicas não serem conclusivas, o grande número de alterações sugestivas, tanto de sequelas como de lesões ativas de tuberculose, confirma a importância da enfermidade na região.

Mesmo considerando a possibilidade de erros diagnósticos e a maior probabilidade de óbitos em adultos e idosos, a identificação de indígenas mediante consulta à variável raça/cor no SINAN mostrou-se útil para identificar desigualdades no adoecimento e morte por tuberculose e revelar que os doentes com idade $\geq 45$ anos apresentaram mais chance de óbito quando comparados aos de 0 a 20 anos. Ainda que a cobertura de tratamento diretamente observado (TDO) tenha sido limitada, as taxas de cura foram elevadas e as de abandono moderadas quando comparadas aos dados nacionais agrupados (22). É admissível pensar que, em São Gabriel da Cachoeira, os casos de tuberculose não tenham sido adequadamente acompa- 
nhados e que a alta do sistema tenha ocorrido ao término do tratamento sem a comprovação por métodos bacteriológicos. Também pode ter havido sobrediagnóstico, ou seja, tratamentos podem ter sido iniciados sem haver-se esgotado a investigação e, portanto, comprovada a presença da tuberculose. Nesse caso, os indígenas classificados equivocadamente como doentes naturalmente evoluiriam para um desfecho favorável.

À semelhança do relatado por outros autores $(23,24)$ em distritos sanitários indígenas de outros estados, no DSEI-ARN podem estar ocorrendo falhas na implantação de rotinas para o correto diagnóstico de tuberculose. Esses distritos podem também estar enfrentando ausência de rotinas sistematizadas para a busca de $\mathrm{SR}$, dificuldades para organização do material utilizado nos exames bacteriológicos, abordagem inadequada do paciente durante a coleta do escarro e insuficiente capacitação dos profissionais envolvidos com essas ações. Além disso, métodos diagnósticos usuais, como PT, cultura de escarro e exame histopatológico foram subutilizados, distanciando-se do recomendado $(1,2)$. Ao que tudo indica, os diagnósticos de tuberculose na região foram baseados apenas em avaliações clínicas e em resultados de radiografias de tórax. Resultados de sorologia para HIV estavam disponíveis apenas para oito pacientes, sendo todos soronegativos.

As falhas apontadas deveriam interferir negativamente nos desfechos dos casos, tornando-os também mais vulneráveis à manutenção da doença e ao desenvolvimento de complicações e formas multi-droga resistentes. No entanto, não parece ter sido esse o caso. As elevadas incidências registradas de 2001 a 2003 podem ser reflexo dos planos estratégicos e emergenciais implantados pelo Ministério da Saúde naquele momento, quando houve intenso processo de descentralização das ações de prevenção, vigilância e controle, assim como aumento da cobertura das notificações no SINAN (10). As ações implementadas podem ter contribuído para redução da transmissão na região, pois, de 2004 a 2007, registrou-se tendência de redução na incidência.

A análise das notificações por localidade mostrou maior concentração da doença em Iauaretê, onde a incidência média no período foi superior à registrada em São Gabriel da Cachoeira, justificando o estudo transversal naquele

TABELA 3. Distribuição de variáveis sociodemográficas, antecedentes clínicos e achados nas radiografias de tórax, de acordo com resultado da prova tuberculínica $\geq 5 \mathrm{~mm}$, Distrito Indígena de lauaretê (AM) Brasil, janeiro de 2010

\begin{tabular}{|c|c|c|c|c|c|}
\hline \multirow[b]{2}{*}{ Variável } & \multirow[b]{2}{*}{ Total } & \multicolumn{3}{|c|}{$\begin{array}{l}\text { Prova tuberculínica } \\
\quad \geq 5 \mathrm{~mm}\end{array}$} & \multirow[b]{2}{*}{$P$-valor ${ }^{a}$} \\
\hline & & No. & $\%$ & $\chi^{2}$ & \\
\hline \multicolumn{6}{|l|}{ Sexo } \\
\hline Masculino & 76 & 59 & 77,6 & 0,19 & 0,665 \\
\hline Feminino & 83 & 62 & 74,7 & & \\
\hline \multicolumn{6}{|l|}{ Idade (anos) } \\
\hline$<15$ & 28 & 5 & 17,9 & 63,39 & $<0,001$ \\
\hline$\geq 15$ & 131 & 116 & 88,5 & & \\
\hline \multicolumn{6}{|l|}{ Escolaridade } \\
\hline Sim & 131 & 101 & 77,1 & 0,48 & 0,488 \\
\hline Não & 20 & 14 & 70,0 & & \\
\hline \multicolumn{6}{|c|}{ Renda mensal (salários mínimos) } \\
\hline$<1$ & 55 & 42 & 76,4 & 0,23 & 0,634 \\
\hline$\geq 1$ & 84 & 67 & 79,8 & & \\
\hline \multicolumn{6}{|l|}{ No. pessoas domicilio } \\
\hline$<6$ & 58 & 46 & 79,3 & 0,63 & 0,429 \\
\hline$\geq 6$ & 91 & 67 & 73,6 & & \\
\hline \multicolumn{6}{|c|}{ Antecedente de tuberculose } \\
\hline Sim & 49 & 48 & 98,0 & 17,81 & $<0,001$ \\
\hline Não & 107 & 72 & 67,3 & & \\
\hline \multicolumn{6}{|c|}{ Contato com tuberculose } \\
\hline Sim & 69 & 57 & 82,6 & 3,26 & 0,071 \\
\hline Não & 87 & 61 & 70,1 & & \\
\hline \multicolumn{6}{|l|}{ Cicatriz vacinal $B C G$} \\
\hline Sim & 135 & 102 & 75,6 & 0,41 & 0,521 \\
\hline Não & 22 & 18 & 81,8 & & \\
\hline \multicolumn{6}{|l|}{ Radiografia de tórax } \\
\hline Com alteração & 29 & 27 & 93,1 & 4,28 & 0,039 \\
\hline Sem alteração & 115 & 87 & 75,7 & & \\
\hline
\end{tabular}

FIGURA 1. Distribuição percentual das reações de enduração $(\mathrm{mm})$ das provas tuberculínicas, Distrito de lauaretê, São Gabriel da Cachoeira (AM), Brasil, 2010ª

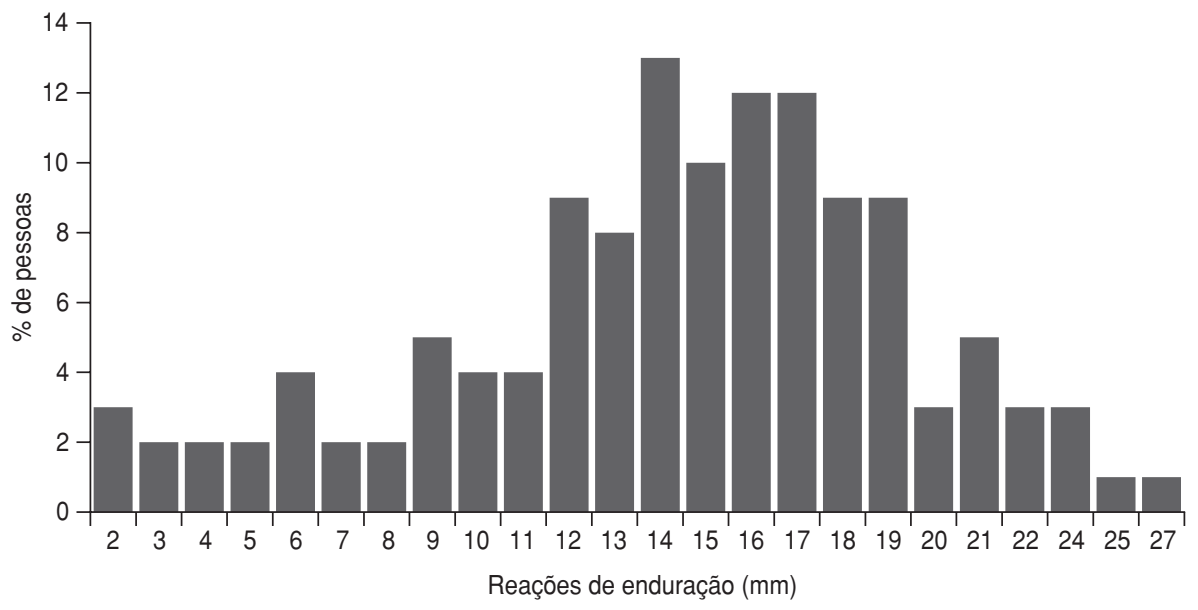

a Total de sujeitos testados: 159; média: $11,6 \mathrm{~mm}$; desvio padrão: 7,4 mm; 19,5\% dos sujeitos apresentaram anergia, ou seja, $\mathrm{PT}=$ zero $\mathrm{mm}$. Os 31 não reatores (zero $\mathrm{mm}$ ) foram omitidos do gráfico por conveniência.

distrito. O cenário encontrado em Iauaretê mostrou-se propício para disseminação de $M$. tuberculosis. Em geral, as habitações investigadas eram pequenas e mal ventiladas, o número de mora- dores por domicílio elevado e a renda mensal baixa (aproximadamente 1 salário mínimo), coerente com a associação entre tuberculose e precárias condições de vida (25). 
TABELA 4. Relatos de 54 indígenas com história anterior de tratamento para tuberculose, Distrito Indígena de lauaretê (AM), Brasil, 2010

\begin{tabular}{lcc}
\hline \multicolumn{1}{c}{ Característica } & No. & $\%$ \\
\hline No. episódios de tuberculose anterior & & \\
1 & 47 & 87,0 \\
2 & 7 & 13,0 \\
Resultado do tratamento anterior & 46 & 85,2 \\
$\quad$ Cura & 6 & 11,1 \\
Abandono & 2 & 3,7 \\
Sem informação & 23 & 42,6 \\
Itinerário terapêutico inicial adotado & 15 & 27,8 \\
$\quad$ Medicamentos da farmácia/posto & 5 & 9,3 \\
Reza & 3 & 5,6 \\
Plantas medicinais/raízes & 8 & 14,8 \\
Ritual xamânico (pajelança) & 13 & 24,1 \\
Sem informação & 12 & 22,2 \\
Explicações para o adoecimento a & 8 & 14,8 \\
Sopro e/ou envenenamento & 2 & 3,7 \\
Contato com doente de tuberculose & 1 & 1,9 \\
Desnutrição/falta de alimentação & 1 & 1,9 \\
Trabalho excessivo & 2 & 3,7 \\
Contato com a etnia Hupdab & 15 & 27,8 \\
Após episódio de gripe ou resfriado & \\
Excesso de bebida alcoólica ou cigarro & & \\
Não sabe informar & & \\
a Respostas espontâneas, obtidas por meio de pergunta aberta aos entrevistados ("você tem alguma explicação \\
para ter adoecido de tuberculose?"). \\
b Na região do estudo, a etnia Hupda é considerada de menor prestígio e poder nas relações inter-tribais e na \\
posse de mercadorias e bens de consumo. Por essa razão, é associada pelas demais etnias à pobreza, à \\
miserabilidade, a posições subalternas e à doença.
\end{tabular}

Não foi possível identificar casos de tuberculose ativa em Iauaretê, sendo a ILTB o desfecho de maior relevância. Mesmo que tenham ocorrido casos em 2010, o delineamento de estudo pode não ter sido capaz de identificá-los oportunamente. À semelhança de outras localidades $(5,26,27)$, em Iauaretê as reações $\geq 5 \mathrm{~mm}$ foram mais frequentes no grupo etário $\geq 15$ anos, com história de tratamento anterior e com imagens radiológicas sugestivas de tuberculose.

A explicação do adoecimento devido a "sopro e/ou envenenamento" e o uso da medicina tradicional indicam a forte presença de fatores culturais associados à tuberculose em Iauaretê. Segundo Buchillet (28), nas sociedades indígenas, o processo saúde-doença não pode ser analisado fora do contexto histórico local, ou seja: é necessário considerar as representações simbólicas e culturais dos indivíduos e as relações por eles estabelecidas entre o mundo natural e sobrenatural. Ao estudar as representações, práticas e demandas de saúde dos Baníwa (etnia do Alto Rio Negro), Garnelo e Wright (29) relatam que os indígenas relacionam a tuberculose à ação de inimigos. Da mesma forma, Welch e Coimbra Jr. (30) descrevem que, entre os Xavante, a feitiçaria é considerada a principal causa de adoecimento por tuberculose. Segundo esses autores, o ato envolve o preparo de um pó de origem vegetal que o agressor deposita em alimentos, bebidas ou objetos pessoais deixados em locais públicos, que possam ser tocados pela vítima, ou então, a cânti$\mathrm{cos} /$ rezas enviadas pelos inimigos com o intuito de causar o mal.

As várias explicações dadas pelos indígenas em Iauaretê para o adoecimento fortalecem o pressuposto da aquisição de um saber fundamentado em relações interétnicas e desvinculado do conceito de transmissibilidade da doença. Esses fatores devem ser considerados pelas equipes multiprofissionais de saúde na elaboração de estratégias de comunicação com os indígenas.

Esta investigação caracterizou-se por analisar dados de uma localidade situada na região da tríplice fronteira BrasilColômbia-Venezuela, na qual reside uma população predominantemente indígena e com alta diversidade étnica. As linhas de fronteira internacional na América Latina foram instituídas somente após o contato com os europeus. Entretanto, a presença ancestral dos povos indígenas nas Américas propiciou laços de parentesco que extrapolam os limites territoriais demarcados pelos não indígenas, ocasionando movimentação intensa desses grupos na região e criando um cenário favorável à transmissão de diversos agravos, dentre os quais destacam-se malária, dengue, hanseníase, parasitoses intestinais e tuberculose (22, 31-33).

Especificamente em relação à tuberculose, Chaparro et al. (34), ao analisarem a situação epidemiológica da doença na Colômbia, destacaram as elevadas incidências nos departamentos fronteiriços com São Gabriel da Cachoeira: Amazonas (92,7/100 000), Vaupés $(70,4 / 100$ 000) e Guainia (60,6/100 000). Ainda na Colômbia, Garcia et al. (35), em investigação no distrito de Mitú (departamento de Vaupés), também relataram que $51,4 \%$ da população era composta por indígenas, sendo que, nesse grupo, foram observadas as maiores proporções de SR e as maiores prevalências de tuberculose.

Como assinalam Braga et al. (36) e Schneider et al. (37), o controle da tuberculose em regiões de fronteira é particularmente difícil, pois não depende somente das estratégias elaboradas por um único país, e sim da integração de todos que possuam fronteiras em comum.

Embora os achados descritos sejam elucidativos da situação da tuberculose em São Gabriel da Cachoeira, é necessário considerar algumas limitações. É possível que tenha havido sub-registro de casos (devido a problemas na cobertura dos serviços ofertados à população) e erros de classificação ou diagnóstico. Lembramos ainda que as análises limitaram-se aos dados disponíveis no SINAN e à forma como as variáveis são estruturadas no sistema. Além disso, no segundo componente, pode ter ocorrido viés de seleção, pois os casos analisados, além de terem sido selecionados de forma não probabilística, podem não ser representativos da experiência de adoecimento em Iauaretê. Em que pese a falta de representatividade estatística dessa amostra de conveniência, entendemos que os dados obtidos retrataram um componente importante das ações de controle empregadas naquela região e forneceram elementos adicionais para aprofundamento da análise dos dados do SINAN.

A despeito das limitações, nossos achados indicam que, embora tenha-se observado redução no número de notificações ao longos dos anos analisados, a transmissão do $M$. tuberculosis foi notável e se manteve em patamares elevados quando 
comparada às médias nacionais e regionais no período estudado (10), submetendo a população a elevado risco de ILTB e tuberculose ativa. Alguns autores sugerem que a escassez de recursos para controlar a tuberculose em populações indígenas, associada à pobreza, à falta de acesso aos serviços de saúde para diagnóstico e tratamento, ao uso frequente de álcool e ao desemprego $(3,6,38)$ pode explicar parcialmente as discrepâncias observadas nos indicadores de incidência e mortalidade entre indígenas e não indígenas. A presença de déficit nutricional, parasitoses intestinais e doenças autoimunes, assim como fatores imunológicos e genéticos, também pode interferir na suscetibilidade à doença $(5,9,39,40)$.

Finalmente, é importante frisar que as estratégias do PNCT tiveram resultados positivos em nível local, onde foi possí- vel verificar altas proporções de cura e tendência de redução na incidência em tempos recentes. Por outro lado, parece haver espaço para melhorar o controle da tuberculose, intensificando a vigilância dos contatos, aprimorando as estratégias de comunicação das equipes com os usuários e inserindo nas investigações componentes que avaliem as condições de vida da população.

Agradecimentos. Os autores agradecem o apoio do grupo de colaboradores e parceiros para o controle da tuberculose em áreas indígenas, especialmente Flávio Pereira Nunes, Coordenador Geral de Atenção à Saúde Indígena e Diretor Substituto do Departamento de Saúde Indígena (DESAI) da Fundação Nacional de Saúde (FUNASA). Agradecem ainda a Perceverando Ribeiro Machado Neto da FUNASA/CORE-RR e ao Moises Palaci e João Batista Pereira da Silva do NDI/UFES e a Luiz Gonzaga Alencar, Adriana Melo Leão, Sizinando Lobato Pontes e todos os profissionais do DSEI-ARN pelo auxílio no trabalho de campo; a Luiz Lopes de Aguiar Neto, Secretário Municipal de Saúde de SGC, Domingos Sávio Borges Barreto, Presidente do Conselho Distrital de Saúde e a todos os indígenas a quem se destinou nosso trabalho.

Financiamento. O estudo teve apoio financeiro do Conselho Nacional de Desenvolvimento Científico e Tecnológico (CNPq) (processo 402505/2008-5) e DESAI/FUNASA.

Conflitos de interesse. Nada declarado pelos autores.

\section{REFERÊNCIAS}

1. World Health Organization (WHO). Global tuberculosis control 2011. WHO Report 2011. Disponível em: http://www.who.int/tb/ publications/global_report/2011/en/index. html Acessado em dezembro de 2012.

2. Brasil, Ministério da Saúde, Secretaria de Vigilância em Saúde, Departamento de Vigilância Epidemiológica. Manual de recomendações para o controle da tuberculose no Brasil. Brasília: Ministério da Saúde; 2011.

3 Nava-Aguilera E, Andersson N, Harris E, Mitchell S, Hamel C, Shea B, et al. Risk factors associated with recent transmission of tuberculosis: systematic review and meta-analysis. Int J Tuberc Lung Dis. 2009;13(1):17-26.

4. Cantwell MF, McKenna MT, McCray E, Onorato IM. Tuberculosis and race/ethnicity in the United States: impact of socioeconomic status. Am J Respir Crit Care Med. 1998;157(4 Pt 1):1016-20.

5. Basta PC, Coimbra CE Jr, Welch JR, Corrêa Alves LC, Santos RV, Bastos Camacho LA. Tuberculosis among the Xavante Indians of the Brazilian Amazon: an epidemiological and ethnographic assessment. Ann Hum Biol. 2010;37(5):643-57.

6. Coimbra CE Jr, Basta PC. The burden of tuberculosis in indigenous people in Amazonia, Brazil. Trans R Soc Trop Med Hyg. 2007; 101(7):635-6.

7. Garnelo L, Brandao LC, Levino A. Dimensões e potencialidades do sistema de informação geográfica na saúde indígena. Rev Saude Publica. 2005;39(4):634-40.

8. Basta PC, Coimbra Junior CE, Escobar AL, Santos RV. Aspectos epidemiológicos da tuberculose na população indígena Suruí, Amazônia, Brasil. Rev Soc Bras Med Trop. 2004;37(4):338-42.

9. Sousa AO, Salem JI, Lee FK, Verçosa MC, Cruaud P, Bloom BR, et al. An epidemic of tuberculosis with a high rate of tuberculin anergy among a population previously unexposed to tuberculosis, the Yanomami Indians of the Brazilian Amazon. Proc Natl Acad Sci USA. 1997;94(24):13227-32.

10. Bierrenbach $A L$, Gomes $A B F$, Noronha EF, Souza MFM. Incidência de tuberculose e taxa de cura, Brasil, 2000 a 2004. Rev Saude Publica. 2007;41 Suppl 1:24-33.

11. Instituto Brasileiro de Geografia e Estatística (IBGE). Sistema IBGE de recuperação automática. Disponível em: http://www.sidra. ibge.gov.br/bda/tabela/protabl.asp?c= $3175 \& z=c d \& o=5 \& i=P$ Acessado em 13 de fevereiro de 2012.

12. Ricardo B, Ricardo F. Povos Indígenas no Brasil: 2006-2010. São Paulo: Instituto Socioambiental (ISA); 2011.

13. Buchillet D, Gazin P. A situação da tuberculose na população indígena do alto Rio Negro (Estado do Amazonas, Brasil). Cad Saude Publica. 1998;14(1):181-5.

14. Levino A, de Oliveira RM. Tuberculose na população indígena de São Gabriel da Cachoeira, Amazonas, Brasil. Cad Saude Publica. 2007;23(7):1728-32.

15. Machado Filho AC. Incidência da tuberculose em indígenas do município de São Gabriel da Cachoeira, AM. Rev Soc Bras Med Trop. 2008; 41(3):243-6.

16. Cabalzar Filho A, Ricardo CA. Povos indígenas do alto e médio Rio Negro: uma introdução à diversidade cultural e ambiental do noroeste da Amazônia brasileira. São Paulo: Instituto Socioambiental/Federação das Organizações Indígenas do Rio Negro/ Ministério da Educação; 1998.

17. Fundação Nacional de Saúde (FUNASA). Disponível em: http://sis.funasa.gov.br/portal/ detalhe_dsei.asp?strcddsei=6 Acessado em 2 fevereiro de 2011.
18. Andrello G. Cidade do índio: transformações e cotidiano em Iauaretê. Rio de Janeiro: UNESP; 2006.

19. IBGE. Série Relatórios Metodológicos, Volume 25. Metodologia do Censo Demográfico 2000. Rio de Janeiro: IBGE; 2003.

20. Arnadottir T, Rieder HL, Trébucq A, Waaler HT. Guidelines for conducting tuberculin skin test surveys in high prevalence countries. Tuber Lung Dis. 1996;77 Suppl 1:1-19.

21. Den Boon S, Bateman ED, Enarson DA, Borgdorff MW, Verver S, Lombard CJ, et al. Development and evaluation of a new chest radiograph reading and recording system for epidemiological surveys of tuberculosis and lung disease. Int J Tuberc Lung Dis. 2005; 9(10):1088-96.

22. Brasil, Ministério da Saúde, Secretaria de Vigilância em Saúde, Sistema Nacional de Vigilância em Saúde. Relatório de situação. Amazonas. Brasília: MS; 2005. Disponível em bvsms.saude.gov.br/bvs/publicacoes/am1. pdf Acessado em 13 de fevereiro de 2012.

23. Nóbrega RG, Nogueira JA, Ruffino-Netto A Duarte de Sá L, Cavalcanti da Silva ATM, Villa TCS. A busca ativa de sintomáticos respiratórios para o controle da tuberculose, no cenário indígena potiguara, Paraíba, Brasil. Rev Lat Am Enfermagem. 2010;18(6):1169-76.

24. Basta PC, Rios DP, Alves LC, Sant'anna CC, Coimbra Junior CE. Estudo clínicoradiológico de crianças e adolescentes indígenas Suruí, Região Amazônica. Rev Soc Bras Med Trop. 2010;43(6):719-22.

25. Ruffino-Neto A. Tuberculose: a calamidade negligenciada. Rev Soc Bras Med Trop. 2002; 35(1):51-8.

26. Escobar AL, Coimbra CE Jr, Camacho LA, Santos RV. Tuberculin reactivity and tuberculosis epidemiology in the Paakanova (Wari') Indians of Rondônia, Southwestern Brazilian 
Amazon. Int J Tuberc Lung Dis. 2004;8(1): 45-51.

27. Basta PC, Coimbra CE Jr, Camacho LA, Santos RV. Risk of tuberculous infection in an indigenous population from Amazonia, Brazil. Int J Tuberc Lung Dis. 2006;10(12):1354-9.

28. Buchillet D. Medicinas tradicionais e medicina oriental na Amazônia. Belém: CEJUP; 1991.

29. Garnelo L, Wright R. Doença, cura e serviços de saúde. Representações, práticas e demandas Baníwa. Cad Saude Publica. 2001;17(2): 273-84.

30. Welch JR, Coimbra Jr CE. Perspectivas culturais sobre transmissão e tratamento da tuberculose entre os Xavánte de Mato Grosso, Brasil. Cad Saude Publica. 2011;27(1): 190-4.

31. Giatti LL, Rocha AA, de Toledo RF, Barreira LP, Rios L, Pelicioni MC, et al. Condições sanitárias e socioambientais em Iauaretê, área indígena em São Gabriel da Cachoeira, AM. Cienc Saude Coletiva. 2007;12(6):1711-23.

32. Imbiriba EN, Basta PC, Pereira ES, Levino A, Garnelo L. Hanseníase em populações indígenas do Amazonas, Brasil: um estudo epidemiológico nos municípios de Autazes, Eirunepé e São Gabriel da Cachoeira (2000 a 2005). Cad Saude Publica. 2009;25(5): 972-84.

33. Bóia MN, Carvalho-Costa FA, Sodré FC, Porras-Pedroza BE, Faria EC, Magalhães GAP, et al. Tuberculose e parasitismo intestinal em população indígena na Amazônia brasileira. Rev Saude Publica. 2009;43(1):176-8.

34. Chaparro PE, Garcia I, Guerrero MI, León CI. Situación de la tuberculosis en Colombia, 2002. Biomédica. 2004;24(Supp 1):102-14.

35. Garcia I, De la Hoz F, Reyes Y, Montoya P, Guerrero MI, León CI. Prevalencia de sintomáticos respiratorios, de infección y enfermedad tuberculosa y factores asociados: estudio basado en población, Mitú, Vaupés, 2001. Biomedica. 2004;24(Supp 1):124-31.

36. Braga JU, Herrero MB, Cuellar CM. Transmissão da tuberculose na tríplice fronteira entre Brasil, Paraguai e Argentina. Cad Saude Publica. 2011;27(7):1271-80.

37. Schneider E, Laserson KF, Wells CD, Moore M. Tuberculosis along the United States-
Mexico border, 1993-2001. Rev Panam Salud Publica. 2004;16(1):23-34.

38. Gessner BD. Incidence rates, clinical features, and case identification of pediatric tuberculosis in Alaska. Int J Tuberc Lung Dis. 1998;2(5): 378-83.

39. Zembrzuski VM, Basta PC, Callegari-Jacques SM, Santos RV, Coimbra CE, Salzano FM, et al. Cytokine genes are associated with tuberculin skin test response in a native Brazilian population. Tuberculosis (Edinb). 2010;90(1):44-9.

40. Zevallos K, Vergara KC, Vergara A, Vidal C, Garcia HH, Evans CA. Tuberculin skin-test reactions are unaffected by the severity of hyperendemic intestinal helminth infections and co-infections. Am J Trop Med Hyg. 2010;83(2): $319-25$.

Manuscrito recebido em 13 de fevereiro de 2012. Aceito em versão revisada em 5 de outubro de 2012

ABSTRACT Objective. To describe the sociodemographic, clinical, and epidemiological characteristics of reported tuberculosis cases among indigenous individuals of São Gabriel de Cachoeira, State of Amazonas, Brazil, and to identify the factors associated with

\section{Tuberculosis in indigenous peoples in the Brazilian Amazon: an epidemiological study in the Upper Rio Negro region}

Key words mortality during treatment; and to estimate the prevalence of latent tuberculosis infection (LTBI) and associated factors and obtain information on the therapeutic course and the individual perceptions regarding acquistion of tuberculosis in the district of Iauaretê.

Methods. Firstly, a retrospective epidemiological study (1997 to 2007) was conducted using data from the Brazilian Notifiable Diseases Surveillance System (SINAN). Next, a cross-sectional study (2010) was conducted with respiratory symptomatic subjects and contacts of Iauaretê.

Results. Seven hundred and twenty-three new cases were reported, with incidence of 273.4/100 000 and mortality of 13.2/100 000. There was a predominance of males $(57 \%)$, aged $\geq 45$ years $(37.6 \%)$, people with no schooling $(42.7 \%)$, and cases from rural areas $(76.9 \%)$. Patients aged 0 to 20 years were at lower risk of death when compared to those aged $\geq 45$ years (OR $=0.3$; IC95\%: 0.1 a 0.9 ). In Iauaretê, with $15.3 \%$ of the reported cases, 184 people were interviewed. A prevalence of LTB of $76.1 \%$ was reported. Tuberculin skin test $\geq 5 \mathrm{~mm}$ was associated with the $\geq 15$-year old age group, history of active tuberculosis, and radiological alterations. A previous history of tuberculosis was cited by 54 people (29.3\%). The main explanation for the disease was "puffing/poisoning" (24.1\%). The therapeutic course included industrialized drugs (42.6\%), medicinal plants/roots, shamanism, and prayer (42.7\%).

Conclusions. The risk of tuberculosis infection and disease in this population was high. Despite the reduced incidence resulting from recent efforts, tuberculosis control requires closer surveillance of contacts and improvement in communication strategies between health teams and indigenous populations.

Tuberculosis; Indians, South American; health of indigenous peoples; epidemiology; health systems; Brazil. 